\title{
Flatness of the Meckel Cave May Cause Primary Trigeminal Neuralgia: a Radiomics-based Study
}

Jinzhi Lin

Zhujiang Hospital

Yong Zhang

Guangdong Second Provincial General Hospital

Wuming Li

Guangdong Second Provincial General Hospital

Jianhao Yan

Guangdong Second Provincial General Hospital

Yiquan Ke ( $\nabla$ kyquan@smu.edu.cn )

Zhujiang Hospital https://orcid.org/0000-0002-7356-8559

Research article

Keywords: flatness, Meckel cave, radiomics, trigeminal neuralgia, cause, morphology

Posted Date: June 23rd, 2021

DOI: https://doi.org/10.21203/rs.3.rs-631679/v1

License: (c) (i) This work is licensed under a Creative Commons Attribution 4.0 International License. Read Full License 


\title{
Flatness of the Meckel cave may cause primary trigeminal neuralgia:
}

\section{a radiomics-based study}

\author{
Jinzhi Lin, ${ }^{1,2 \dagger}$ Yong Zhang, ${ }^{2, \dagger}$ Wuming $\mathrm{Li},{ }^{2}$ Jianhao Yan, ${ }^{2}$ Yiquan $\mathrm{Ke}^{1}$ \\ ${ }^{\dagger}$ These authors contributed equally to this work.
}

1 The National Key Clinical Specialty, The Engineering Technology Research Center of Education Ministry of China, Guangdong Provincial Key Laboratory on Brain Function Repair and Regeneration, Department of Neurosurgery, Zhujiang Hospital, Southern Medical University, Guangzhou 510282, China

2 Department of Neurosurgery, Guangdong Second Provincial General Hospital, Guangzhou, 510317, China

Corresponding author: Yiquan Ke

Email:kyquan@smu.edu.cn

Key word: flatness, Meckel cave, radiomics, primary trigeminal neuralgia, morphology

\begin{abstract}
Background: Neurovascular contact (NVC) is the main cause of primary trigeminal neuralgia (PTN); however, cases of PTN without NVC are still observed. In this study, the Meckel cave (MC) morphology in PTN were analyzed by radiomics and compared to healthy controls (HCs) to explore the cause of PTN.
\end{abstract}

Methods: We studied the 3.0T MRI data of 115 patients with PTN and 46 HCs. Bilateral MC was modeled using the 3Dslicer software, and the morphological characteristics of $\mathrm{MC}$ were 
analyzed using the radiomics method.

Results: The right side incidence rate in the PTN group was higher than the left side incidence. By analyzing the flatness feature of MC, we observed that the affected side of the PTN was lower than that of the unaffected side, the right MC of the PTN and $\mathrm{HC}$ was lower than that of the left MC, the MC of the affected side of the left and right PTN without bilateral NVC was lower than that of the unaffected side.

Conclusions: By providing a method to analyze the morphology of $\mathrm{MC}$, we found that there is asymmetry in the morphology of bilateral MC in the PTN and HC groups. It can be inferred that the flatness of MC may be a cause of PTN.

Key word: flatness, Meckel cave, radiomics, trigeminal neuralgia, cause

\section{Key points:}

Flatness of the Meckel cave may be a cause of primary trigeminal neuralgia.

There is asymmetry in the morphology of bilateral Meckel cave of primary trigeminal neuralgia and healthy controls.

The shape features of the Meckel cave can be accurately quantified and analyzed by radiomics method.

\section{Introduction}

Trigeminal neuralgia (TN) is a chronic neuropathic pain disorder ${ }^{1}$, that can lead to poor quality of life and even suicide ${ }^{2}$. TN can be classified as primary TN (PTN), which is either 
classical or idiopathic, depending on the degree of neurovascular contact (NVC), or as secondary $\mathrm{TN}$, which is caused by pathology rather than $\mathrm{NVC}^{3}$. Asymptomatic $\mathrm{NVC}$ in patients with $\mathrm{PTN}^{4}$ and high prevalence of NVC in individuals without $\mathrm{TN}^{5}$ suggest that $\mathrm{NVC}$ is not the only pathogeny of PTN, which is worthy of further study.

Anatomically, the intracranial trigeminal nerve is divided into the root entry zone (REZ) and the Meckel cave (MC) segments after emerging from the brain stem. Many studies ${ }^{1-3}$ have proven that morphological changes (atrophy or displacement) with NVC of the trigeminal nerve in the REZ are related to symptomatic PTN. However, no significant correlation has been observed between PTN and NVC without morphological changes. Some currently unknown etiological factors are likely to play an important role ${ }^{6}$. The MC, as an important structure after the REZ of the trigeminal nerve, is hence worth studying.

Previous studies on the trigeminal nerve morphology of PTN have focused on the REZ and lacked emphasis on the MC. The MC is an important part of the trigeminal nerve after the REZ, which is composed of numerous small fibers and the trigeminal ganglion consisting of a small amount of solid tissue ${ }^{7}$. Some cases $^{8,9}$ report that ipsilateral hypoplastic MC, which may lead to morphological atrophy, as well as crowded MC, may have produced symptoms consistent with TN. Percutaneous balloon compression (PBC) can effectively relieve the pain symptoms of patients with PTN by changing the shape of the MC, which expands after balloon dilation; moreover, the balloon shape is a parameter that has a very strong impact on outcome $^{10,11}$. Therefore, it can be inferred that the morphological characteristics of MCs may be related to PTN.

Therefore, we hypothesized that MCs with narrow morphological characteristics are 
related to PTN. MRI provides the best way to evaluate the morphological characteristics of $\mathrm{PTN}^{6}$ and radiomics can automatically quantify the phenotypic characteristics of medical imaging $^{12}$. The aim of the present study was to collect 3.0T MRI data of patients with PTN and of healthy controls (HCs) to accurately model the bilateral MC with the 3Dslicer software, and then analyze the MC morphology using the radiomics method to explore the cause of PTN.

\section{Materials and Methods}

\section{Data Source}

The study was approved by the Medical Ethics Committee of the Guangdong Second Provincial General Hospital in Guangzhou, China. Written informed consent was obtained from each subject. From January 2018 to January 2020, 115 PTN patients (mean age: $62.89 \pm$ 10.43 years, 70 female) were recruited from the Department of Neurosurgery of the Second Provincial General Hospital of Guangdong. The PTN diagnosis was based on the Third edition of International Classification of Headache Disorders ${ }^{13}$. Exclusion criteria were secondary TN, other primary headache disorders (e.g., migraine or tension-type headache) and past surgical treatment of PTN (such as microvascular decompression, PBC, and

radiofrequency). We also recruited 46 healthy volunteers as HC (mean age: $49.28 \pm 10.96$ years, 31 female). Table 1 lists the demographics and patient characteristics.

\section{MR imaging}

MRI examinations were performed by a Philips Achieva 3.0T dual-source MRI scanner (Philips Healthcare, Amsterdam, The Netherlands). Balanced turbo field echo (BTFE) and 
enhanced T1 high-resolution isotropic volume excitation (ETHRIVE) sequences were obtained using a fast field echo (FFE) pulse sequence with repetition time (TR) $=25 \mathrm{~ms}$, echo time $(\mathrm{TE})=4.1 \mathrm{~ms}$, flip angle $(\mathrm{FA})=30^{\circ}$, acquisition matrix $=256 \times 256$, and slice thickness $=$ $1.0 \mathrm{~mm}$.

NVC was defined on the analysis of imaging with no perceptible CSF signal intervening between the vascular structure and the cisternal segment of the trigeminal nerve. All MRI studies were reviewed and analysed by an experienced neuroradiologist and a neurosurgeon who were blinded to the diagnosis and the laterality of the headache. When discrepancies existed, the two assessors reached at a consensus for statistical analysis.

\section{Image Processing}

The dcm2niigui (http://people.cas.sc.edu/rorden/mricron/stats.html) was used to convert the original raw digital imaging and communications (DICOM) in medicine data of head MRA ETHRIVE and BTFE sequences into nifti (Neuroimaging Informatics Technology Initiative) format. Images were registered and fused using Co-register (Estimate \& Re-slice) of the SPM12 (http://www.fil.ion.ucl.ac.uk/spm/software/spm12/) and opened by the 3Dslicer software (v4.11, https://www.slicer.org/) $)^{14}$.

The segment-editor function of the 3Dslicer software was used to model and correct the bilateral MC in BTFE sequence through axial, coronal, and sagittal planes, and then registered ETHRIVE sequence was opened to overlap the BTFE sequence. At last, we observed the MC wall with ETHRIVE sequence, determined the edge of the MC from the axial, coronal, and sagittal planes, and recalibrated the edge of the MC again. (Figure 1) 


\section{Morphological features acquisition}

Through the radiomics (revision:3b2c531) plug-in of the 3Dslicer software, we analyzed bilateral $322 \mathrm{MCs}$ and got 13 morphological features of shape ${ }^{15}$ including:

1. Voxel volume (using the following formula:

$$
V_{\text {voxel }}=\sum_{k=1}^{N_{v}} V_{k}
$$

where the volume of the $M C \mathrm{~V}_{\text {voxel }}$ is multiplying the number of voxels in the region of interest (ROI) by the volume of a single voxel $\mathrm{V}_{\mathrm{k}}$.),

2. Surface area (using the following formula:

$$
\begin{array}{r}
A_{i}=\frac{1}{2}\left|a_{i} b_{i} \times a_{i} c_{i}\right| \\
A=\sum_{i=1}^{N_{f}} A_{i}
\end{array}
$$

where $\mathrm{a}_{\mathrm{i}} \mathrm{b}_{\mathrm{i}}$ and $\mathrm{a}_{\mathrm{i}} \mathrm{c}_{\mathrm{i}}$ are edges of the $\mathrm{i}^{\text {th }}$ triangle in the mesh, formed by vertices $\mathrm{a}_{\mathrm{i}}, \mathrm{b}_{\mathrm{i}}$ and $\mathrm{c}_{\mathrm{i}}$. To calculate the surface area, first the surface area $A_{i}$ of each triangle in the mesh is calculated (1). The total surface area is then obtained by taking the sum of all calculated subareas (2).),

3. Maximum 3D diameter (is defined as the largest pairwise Euclidean distance between ROI surface mesh vertices. Also known as Feret diameter.),

4. Maximum 2D diameter slice (is defined as the largest pairwise Euclidean distance between ROI surface mesh vertices in the row-column (generally the axial) plane.),

5. Maximum 2D diameter column (is defined as the largest pairwise Euclidean distance between ROI surface mesh vertices in the row-slice (usually the coronal) plane.),

6. Maximum 2D diameter row (is defined as the largest pairwise Euclidean distance between ROI surface mesh vertices in the column-slice (usually the sagittal) plane.), 
7. Minor axis length (using the following formula:

$$
\text { least axis }=4 \sqrt{\lambda_{\text {least }}}
$$

This feature yield the smallest axis length of the MC-enclosing ellipsoid and is calculated using the largest principal component $\lambda_{\text {least. }}$ In case of a $2 \mathrm{D}$ segmentation, this value will be 0 .),

8. Major axis length (using the following formula:

$$
\text { major axis }=4 \sqrt{\lambda \text { major }}
$$

This feature yield the largest axis length of the ROI-enclosing ellipsoid and is calculated using the largest principal component $\lambda_{\text {major. }}$ ),

9. Least axis length (using the following formula:

$$
\text { least axis }=4 \sqrt{\lambda_{\text {least }}}
$$

This feature yield the smallest axis length of the ROI-enclosing ellipsoid and is calculated using the largest principal component $\lambda_{\text {least. }}$ In case of a $2 \mathrm{D}$ segmentation, this value will be 0 .),

10. Flatness (using the following formula:

$$
\text { Flatness }=\sqrt{\frac{\lambda_{\text {least }}}{\lambda_{\text {major }}}}
$$

Flatness shows the relationship between the largest and smallest principal components in the MC shape. Here, $\lambda_{\text {major }}$ and $\lambda_{\text {least }}$ are the lengths of the largest and smallest principal component axes. The values range between 1 (non-flat, sphere-like) and 0 (a flat object, or single-slice segmentation).

The model of the MC was independently performed by two neurosurgeons who were proficient in using the 3Dslicer software, and the morphological data were averaged.

\section{Statistical Analysis}


Independent sample $\mathrm{T}$ tests were performed to assess differences in gender, age; Binomial test was used to test the left and right sides of PTN; Linear regression analysis were used to assess differences in morphological features of the MC (voxel volume, surface area, maximum 3D diameter, maximum 2D diameter slice, maximum 2D diameter column, maximum 2D diameter row, minor axis length, major axis length, least axis length, flatness); Paired sample T test were used to assess differences in morphological features of the bilateral MC of two groups respectively; and Kendall correlation analysis were used to assess correlation in the morphological features of the MC in PTN and HC groups using IBM SPSS Version 22.0 (IBM Corp., Armonk, New York, USA). $P<0.05$ was considered statistically significant.

\section{Results}

Differences of demographic characteristics and morphological features of the MC between PTN and HC groups.

The demographic characteristics of PTN and HC groups and the 10 morphological features of 322 MCs were analyzed. The right side incidence rate in the PTN group was higher than the left side incidence $(66.96 \%$ vs $33.04 \%, P<0.01)$ (Table 1).

The voxel volume of the MC ranged $94.23 \sim 1589.90 \mathrm{~mm}^{3}$, the surface area ranged 131.16 958.39 $\mathrm{mm}^{2}$ (Figure 2), the maximum 3D diameter ranged $8.42 \sim 19.70 \mathrm{~mm}$, the maximum 2D diameter slice ranged 7.28 16.88mm, the maximum 2D diameter column ranged $7.37 \sim 19.10 \mathrm{~mm}$, the maximum $2 \mathrm{D}$ diameter row ranged $8.03-19.64 \mathrm{~mm}$, the minor axis length ranged $6.50 \sim 12.46 \mathrm{~mm}$, the major axis length ranged $7.32 \sim 18.46 \mathrm{~mm}$, the least axis length 
ranged 2.84 5.98mm, there was no significant difference between PTN and HC groups (Table 2).

By analyzing the flatness feature of the $\mathrm{MC}$, we found that the flatness of $\mathrm{MC}$ ranged from $0.25 \sim 0.54$, the affected side of the PTN was lower than that of the unaffected side $(P<0.05)$, the right MC of HCs and patients with PTN was lower than left MC $(P<0.01)$. (Figure 2) (Table 2).

Differences in morphological features of bilateral MC in PTN and HC groups.

By analysing the morphological features of $\mathrm{MC}$ in the $\mathrm{HC}$, we found that in some features, the left side and right side were significantly different. These differences included the maximum 3D diameter $(P<0.05)$, maximum 2D diameter column $(P<0.05)$, least axis length $(P<0.05)$, and flatness $(P<0.01)$ (Table 2).

As for the morphological characteristics of MC in PTN, we also found that there were significant differences between the left side and the right side in some features. These differences included the surface area $(P<0.01)$, maximum 3D diameter $(P<0.01)$, maximum 2D diameter slice $(P<0.01)$, maximum 2D diameter column $(P<0.01)$, maximum 2D diameter row $(P<0.05)$, minor axis length $(P<0.01)$, major axis length $(P<0.01)$, least axis length $(P$ $<0.05)$, and flatness $(P<0.01)$ (Table 2).

Correlation analysis of MC Flatness features in PTN and HC groups.

The flatness of $\mathrm{HC}$ was positively correlated with the least axis length $(r=0.325, P<0.01)$ and negatively correlated with the major axis length $(r=-0.263, P<0.01)$, maximum 3D 
diameter $(r=-0.217, P<0.01)$, maximum 2D diameter slice $(r=-0.164, P<0.05)$, maximum 2D diameter row $(r=-0.169, P<0.05)$. The most significant correlation was least axis length, followed by major axis length.

The flatness of PTN was positively correlated with the least axis length $(r=0.351, P<0.01)$, major axis length $(r=-0.238, P<0.01)$, and negatively correlated with the maximum 3D diameter $(r=-0.210, P<0.01)$, maximum 2D diameter slice $(r=-0.152, P<0.01)$, maximum 2D diameter column $(r=-0.109, P<0.05)$ and maximum 2D diameter row $(r=-0.147, P<$ 0.05). The most significant correlation was least axis length, followed by major axis length (Figure 3).

Differences in morphological features of the MC in PTN group without bilateral NVC.

Table 3 summarizes the NVC of PTN group. There were 9 patients with left PTN and 9 patients with right PTN without bilateral NVC. The flatness of MC of the left PTN on the affected side was lower than that of the unaffected side $(P<0.051)$, the flatness of MC of right PTN on the affected side was lower than that of the unaffected side $(P<0.05)$. (Table 4) (Figure 4) Other morphological features were no significant differences.

\section{Discussion}

The MC is an important anatomical part of the intracranial trigeminal nerve after REZ. It is the dural space formed by the trigeminal nerve root, which is enclosed by the dural membrane of the posterior fossa in the middle fossa and crosses the rocky ridge; moreover, it contains the 
trigeminal ganglion and the trigeminal cistern ${ }^{16}$. Morphological changes in the $\mathrm{MC}$ are very important in the diagnosis ${ }^{17-22}$ and treatment ${ }^{23,24}$ of intracranial diseases, such as radiofrequency thermocoagulation and PBC of PTN ${ }^{25-29}$. Some $\operatorname{cases}^{8,9}$ report that ipsilateral hypoplastic MC, which may lead to morphological atrophy, as well as crowded MC, may have produced symptoms consistent with TN. At present, there is little research on the morphology of MC in patients with PTN and in healthy people, and there is also a lack of statistical data comparing the MC morphology between these groups.

In this study, we introduced the method of the MC morphological features extraction based on radiomics. In the identification of MC anatomy by MRI, most studies ${ }^{8,17-19}$ only use one of the $\mathrm{T} 1$ or $\mathrm{T} 2$ sequences. $\mathrm{T} 1$ enhanced sequence is helpful to determine the wall of the $\mathrm{MC}^{30}$, but lacks sensitivity of cerebrospinal fluid and nerves in the MC. T2 sequence can distinguish the MC content sensitively, but cannot display the wall of the MC clear enough. We used the fusion of ETHRIVE and BTFE sequences of 3.0T MRI to model MC, which can clearly show not only the structure in the MC, but also the MC wall.

Radiomics is an automatic algorithm to quantify the phenotypic characteristics of medical imaging, which can be used after installing PyRadiomics plug-in through the 3Dslicer software $^{12}$ and is widely used in medical science research ${ }^{31-34}$. In this study, we obtained morphological features of the MC by radiomics, which can be divided into two categories. One is the feature describing the size of $\mathrm{MC}$, including voxel volume, surface area, maximum 3D diameter, maximum 2D diameter slice, maximum 2D diameter column, maximum 2D diameter row, minor axis length, major axis length, and least axis length. The other is the feature describing the shape features of MC, including sphericity and flatness. 
The morphological results (voxel volume and surface area) of MC in this study were significantly higher than those of an autopsy ${ }^{35}$, which is due to the loss of MC cerebrospinal fluid in the cadaver head as well as the fixation of the corpse.

The precise acquisition of MC morphological data through the radiomics method is beneficial for the preparation of PBC for PTN. PBC requires the puncture of the MC and filling up of the balloon to compress the semilunar ganglion nerve $\mathrm{e}^{36}$. The accurate evaluation of the $\mathrm{MC}$ volume before $\mathrm{PBC}$ can not only provide reference for the balloon filling volume, but also prepare appropriate balloon volume for some special MC volumes (the maximum volume of MC in this study was $1589.90 \mathrm{~mm} 3)$.

The shape analysis of MC is difficult to quantify and is generally described as an ellipse or open-ended three-fingered glove ${ }^{37}$. By contrast, flatness was obtained in this study using radiomics, and was accurately quantified and analyzed.

In the HC group, the maximum 3D diameter and maximum 2D diameter column of the right $\mathrm{MC}$ were greater than those of the left $\mathrm{MC}(\mathrm{P}<0.05)$, whereas the least axis length and flatness were less than those of the left MC $(\mathrm{P}<0.05)$. In the PTN group, the surface area, maximum 3D diameter, maximum 2D diameter slice, maximum 2D diameter column, maximum 2D diameter row, minor axis length, and major axis length of the right $\mathrm{MC}$ were greater than those of the left $\mathrm{MC}(\mathrm{P}<0.05)$, whereas the least axis length and flatness were less than those of the left MC $(\mathrm{P}<0.05)$, suggesting that the shape of the bilateral MC was asymmetric. The MC on the right side is flatter and larger than that on the left side, especially in the PTN group. To our knowledge, the bilateral asymmetry of MC has been rarely described in the literature among healthy people and patients with PTN. There is an asymmetry between 
the two hemispheres of the human brain, including the cortical projection area associated with the trigeminal nerve (such as the posterior central gyrus, cingulate gyrus, and paracentral gyrus $)^{38-40}$. Whether the asymmetry of the MC shape is related to the volume or thickness of the cerebral cortex deserves further study.

The complicated pathogenesis of PTN has always been a focus and is unclear, to a large extent, in medical knowledge. Although the most common pathogenic mechanism is NVC in the $\mathrm{REZ}^{41}$, asymptomatic vessel-nerve contact is present in $92 \%$ of patients, and there is no NVC in $3.1 \%-17 \%$ of $\mathrm{PTN}^{4}$. Therefore, this theory is not applicable to all the cases ${ }^{42}$.

By analyzing the flatness features of MC, we found that the affected side of the PTN was lower than that of the unaffected side $(P<0.05)$, and the affected MC side of the left PTN without bilateral NVC was lower than that of the unaffected side $(P<0.051)$. Moreover, the affected MC side of the right PTN without bilateral NVC was lower than that of the unaffected side $(P<0.05)$. These results suggest that flatness was still associated with PTN even after NVC exclusion. A further analysis found that the right MC of $\mathrm{HCs}$ and patients with PTN was lower than that of the left MC $(P<0.01)$, and that the prevalence of PTN on the right side was higher than that on the left side $(P<0.01)$, which was consistent with the literature ${ }^{2,43}$. Our current study suggests that this finding is more than a mere chance association, and that the flatness of the MC may be associated with PTN and is likely to explain the high prevalence of right PTN.

Based on the results of this study, we found that the right MC is larger and flatter than the left MC, which may lead to increased density or compression of the trigeminal nerve at the right MC. This compression of the trigeminal nerve can lead to PTN. Ipsilateral MC dysplasia 
may produce symptoms consistent with $\mathrm{TN}^{8}$ and crowded $\mathrm{MC}$ may also lead to $\mathrm{TN}^{9}$, Therefore, we believe that the flatness of the MC may be a cause of PTN.

The deficiency of this study is that the $P$ value of the MC flatness results on the affected side MC of the left PTN without bilateral NVC were 0.0509 . Despite being very close, it did not reach the statistical level of significance $(P<0.05)$, which was related to the insufficient sample size of this study. We also only analyzed the morphological characteristics of MCs without further analysis of the trigeminal nerve in MC. The next step is to increase the number of samples and analyze the trigeminal nerve components in the MC to explore the etiology of PTN.

\section{Conclusions}

By providing a method to analyze the morphology of $\mathrm{MC}$, we found that there is asymmetry in the morphology of bilateral MC in the PTN and HC groups. It can be inferred that the flatness of MC may be a cause of PTN.

\section{References}

1. Bendtsen L, Zakrzewska JM, Heinskou TB, et al. Advances in diagnosis, classification, pathophysiology, and management of trigeminal neuralgia. Lancet Neurol. 2020;19(9):784-796.

2. Cruccu G, Di Stefano G, Truini A. Trigeminal Neuralgia. N Engl J Med. 2020;383(8):754-762.

3. EAN guideline on trigeminal neuralgia. European Journal of Neurology. 2019;

4. Peker S, Dinçer A, Necmettin Pamir M. Vascular compression of the trigeminal nerve is a 
frequent finding in asymptomatic individuals: 3-T M R imaging of 200 trigeminal nerves using 3D CISS sequences. Acta Neurochir (Wien). 2009;151(9):1081-8.

5. Selçuk P, Özlem K, İbrahim Ü, Necmettin PM. Microanatomy of the central myelin-peripheral myelin transition zone of the trigeminal nerve. Neurosurgery. 2006;(2):354-9.

6. Bendtsen L, Zakrzewska JM, Abbott J, al. e. European Academy of Neurology guideline on trigeminal neuralgia. European journal of neurology. 2019;26:831-49.

7. Rubinstein D, Stears R, Stears J. Trigeminal nerve and ganglion in the Meckel cave: appearance at CT and MR imaging. Radiology. 1994;193(1):155-159.

8. Cleary DR, Handwerker J, Ansari H, Ben-Haim S. Three Cases of Trigeminal Neuralgia with Radiographic Absence of Meckel's Cave. Stereotact Funct Neurosurg. 2019;97(4):249-254.

9. Panda A, Lehman V, Garza I, Diehn F. Trigeminal Neuralgia Due to Meckel's Cave Crowding in the Setting of Possible Idiopathic Intracranial Hypertension without Papilledema. Annals of Indian Academy of Neurology. 2020;

10. Konstantinos K, Gastón S, Göran L, Jaleh W, Bengt L. Factors that influence outcome of percutaneous balloon compression in the treatment of trigeminal neuralgia. Neurosurgery. 2010;(4):925-34; .

11. Scranton RA, Shah K, Cohen-Gadol AA. Alternative customized instrumentation and technique for percutaneous balloon compression rhizotomy for trigeminal neuralgia. Journal of Neurosurgery. 2019;132(6):1-4.

12. van Griethuysen JJM, Fedorov A, Parmar C, et al. Computational Radiomics System to Decode the Radiographic Phenotype. Cancer Res. 2017;77(21):e104-e107.

13. Headache Classification Committee of the International Headache Society (IHS) The International 
Class ification of Headache Disorders, 3rd edition. Cephalalgia. 2018;38(1):1-211.

14. Lin J, Zhang Y, Peng R, et al. Preoperative imaging and microscopic navigation during surgery can avoid unnecessarily opening the mastoid air cells through craniotomy using the retrosigmoid approach. World Neurosurgery. 2019;121:e15-e21.

15. Zwanenburg A, Leger S, Vallières M, Lck S. Image biomarker standardisation initiative. arXiv eprints. 2016:arXIv:1612.07003;

16. Rigante L, Herlan S, Tatagiba MS, Stanojevic M, Hirt B, Ebner FH. Petrosectomy and Topographical Anatomy in Traditional Kawase and Posterior Intradural Petrous Apicect omy (PIPA) Approach: An Anatomical Study. World Neurosurg. 2016;86:93-102.

17. Wright J, Wycoco V. Asymmetric Meckel cave enlargement: a potential marker of PHACES syndrome. American Journal of Neuroradiology. 2017;38(6):1223-1227.

18. Kamali A, Sullivan K, Rahmani F, et al. Indentation and Transverse Diameter of the Meckel Cave: Imaging Markers to Diagnose Idiopathic Intracranial Hypertension. American Journal of Neuroradiology. 2020;41(8):1487-1494.

19. Aaron GP, Illing E, Lambertsen Z, et al. Enlargement of Meckel's cave in patients with spontaneous cerebrospinal fluid leaks. Int Forum Allergy Rhinol. 2017;7(4):421-424.

20. Moisak GI, Matsko DE, Chernov SV, et al. What Tactics Should a Surgeon Choose to Treat a Black Extracerebral Tumor? A Case Report of Psammomatous Melanotic Schwannoma of Meckel's Cave and Literature Review. World neurosurgery. 2018;116:337-342.

21. Taghipour M, Saffarrian A, Ghaffarpasand F, Azarpira N. Dumbbell-Shape Hydatid Cyst of Meckel Cave Extending to Cerebellopontine Angle and Middle Fossa; Surgical Technique and Outcome of Rare Case. World neurosurgery. 2017;104:1049. e7-1049. e10. 
22. Strub GM, Moore JE, Huang AT, Stevenson AW, Reiter ER. Chronic facial pain and Meckel cave masses as the initial presentation of neurosarcoidosis: a case report. ENT: Ear, Nose \& Throat Journal. 2013;92(12):558-65.

23. Truong HQ, Sun X, Celtikci E, et al. Endoscopic anterior transmaxillary "transalisphenoid" approach to Meckel's cave and the middle crania 1 fossa: an anatomical study and clinical application. $J$ Neurosurg. 2018;130(1):227-237.

24. Hughes JD, Kapurch J, Van Gompel JJ, et al. Diagnosis and Outcome of Biopsies of Indeterminate Lesions of the Cavernous Sinus and Meckel's Cave: A Retrospective Case Series in 85 Patients. Neurosurgery. 2018;83(3):529-539.

25. Bond JD, Xu Z, Zhang H, Zhang M. Meckel's Cave and Somatotopy of the Trigeminal Ganglion. World Neurosurg. 2021;148:178-187.

26. Linjingzhi, Zhangyang, Penruoyu, et al. Double targets percutaneous radiofrequency thermocoagulation in treatment of primary trigeminal neuralgia. Chinese Journal of Neuromedicine. 2017;5(16):508-512.

27. Linjingzhi, Wangmo, Luoweishi, et al. Cardiovascular responses during stereotactic assisted percutaneous radiofrequency thermocoagulation therapy for primary trigeminal neuralgia. Chinese Journal of Neurosurgical Disease Research. 2014;13(1):67-71.

28. Linjingzhi, Zhuminghua, Huangkaimin, Zhangyang, Zhangyong. Clinical research of eight areas of trigeminal nerves with electrical slimalation. Chinese Journal of Neuromedicine.

2015;14(012):1271-1274.

29. Lin J, Zhang y, Lu J, et al. Near field potential of trigeminal nerve entering pontine area.

Guangdong Medical Journal. 2020;14(41):1432-1436. 
30. Tang Z, Feng X, Qian W, Song J. Evaluation of magnetic resonance imaging criteria for Meckel's cave lesion: logistic regression analy sis and correlation with surgical findings. Clin Imaging. $2011 ; 35(5): 329-35$.

31. Bailly C, Bodet-Milin C, Bourgeois M, et al. Exploring Tumor Heterogeneity Using PET Imaging: The Big Picture. Cancers (Basel). 2019;11(9)

32. Huang YQ, Liang CH, He L, et al. Development and Validation of a Radiomics Nomogram for Preoperative Prediction of Lymph Node Metastas is in Colorectal Cancer. J Clin Oncol.

2016;34(18):2157-64.

33. Lambin P, Leijenaar RTH, Deist TM, et al. Radiomics: the bridge between medical imaging and personalized medicine. Nat Rev Clin Oncol. 2017;14(12):749-762.

34. Sun R, Limkin EJ, Vakalopoulou M, et al. A radiomics approach to assess tumour-infiltrating CD8 cells and response to anti-PD-1 or anti-PD-L1 immunotherapy: an imaging biomarker, retrospective multicohort study. Lancet Oncol. 2018;19(9):1180-1191.

35. Sabanc PA, Batay F, Civelek E, et al. Meckel's cave. World Neurosurg. 2011;76(3-4):335-41; .

36. Sterman-Neto H, Fukuda CY, Duarte KP, et al. Balloon compression vs radiofrequency for primary trigeminal neuralgia: a randomized, controlled tria 1. Pain. 2021;162(3):919-929.

37. Malhotra A, Tu L, Kalra VB, et al. Neuroimaging of Meckel's cave in normal and disease conditions. Insights Imaging. 2018;9(4):499-510.

38. Liang X, Zhao C, Jin X, et al. Sex-related human brain asymmetry in hemispheric functional gradients. NeuroImage. 2021;229:117761.

39. Shuwei L, Yunxia L, Yuchun T. The construction, asymmetry and genetic correlation of 4D digital brain atlas. JOURNAL OF SHANDONG UNIVERSITY(HEALTH SCIENCES). 2010;51(1):33-41. 
40. Guadalupe T, Kong X-Z, Akkermans SE, Fisher SE, Francks C. Relations between hemispheric asymmetries of grey matter and auditory processing of spoken syllables in 281 healthy adults. Brain Structure and Function. 2021;(4):1-12.

41. Love S, Coakham HB. Trigeminal neuralgia: pathology and pathogenesis. Brain. 2001;124(Pt 12):2347-60.

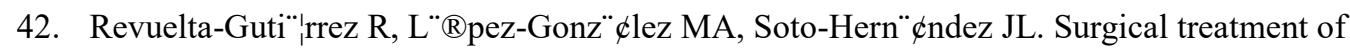
trigeminal neuralgia without vascular compression: 20 years of experience. Surg Neurol. $2006 ; 66(1): 32-6$

43. Giorgio L, Khadija R, Emer O, et al. Trigeminal neurovascular contact in SUNCT and SUNA: a cross-sectional magnetic resonance study. Brain. 2020;143(12):3619-3628.

List of abbreviations: HC, healthy controls; MC, Meckel cave; NVC, neurovascular contact; PBC, percutaneous balloon compression; PTN, primary trigeminal neuralgia; REZ, root entry zone; TN, trigeminal neuralgia; BTFE, balanced turbo field echo; ETHRIVE, enhanced T1 high-resolution isotropic volume excitation; ROI, Region of interest

\section{Declarations:}

\section{- Ethics approval and consent to participate}

The study was approved by the Medical Ethics Committee of the Guangdong Second Provincial General Hospital in Guangzhou, China. Written informed consent was obtained from each subject. 


\section{- Consent for publication}

The scientific guarantor of this publication is Yiquan Ke.

\section{- Availability of data and materials}

The data that support the findings of this study are available from the corresponding author, upon reasonable request.

\section{- Competing interests}

The authors declare that they have no competing interests.

\section{- $\quad$ Funding}

Yiquan Ke received funding from the Natural Science Foundation of China (No. 81772651) Jinzhi Lin received funding from the Medical Scientific Research Foundation of Guangdong Province (No. A2019399)

\section{- Authors' contributions}

Jinzhi Lin: data collection, data analysis and interpretation, writing and revising the manuscript. Yong Zhang, Wuming Li, Jianhao Yan: data collection, writing and revising the manuscript. Yiquan Ke: drafting of the study, writing and revising the manuscript. The author(s) read and approved the final manuscript.

\section{- Acknowledgements}

None 

Figures

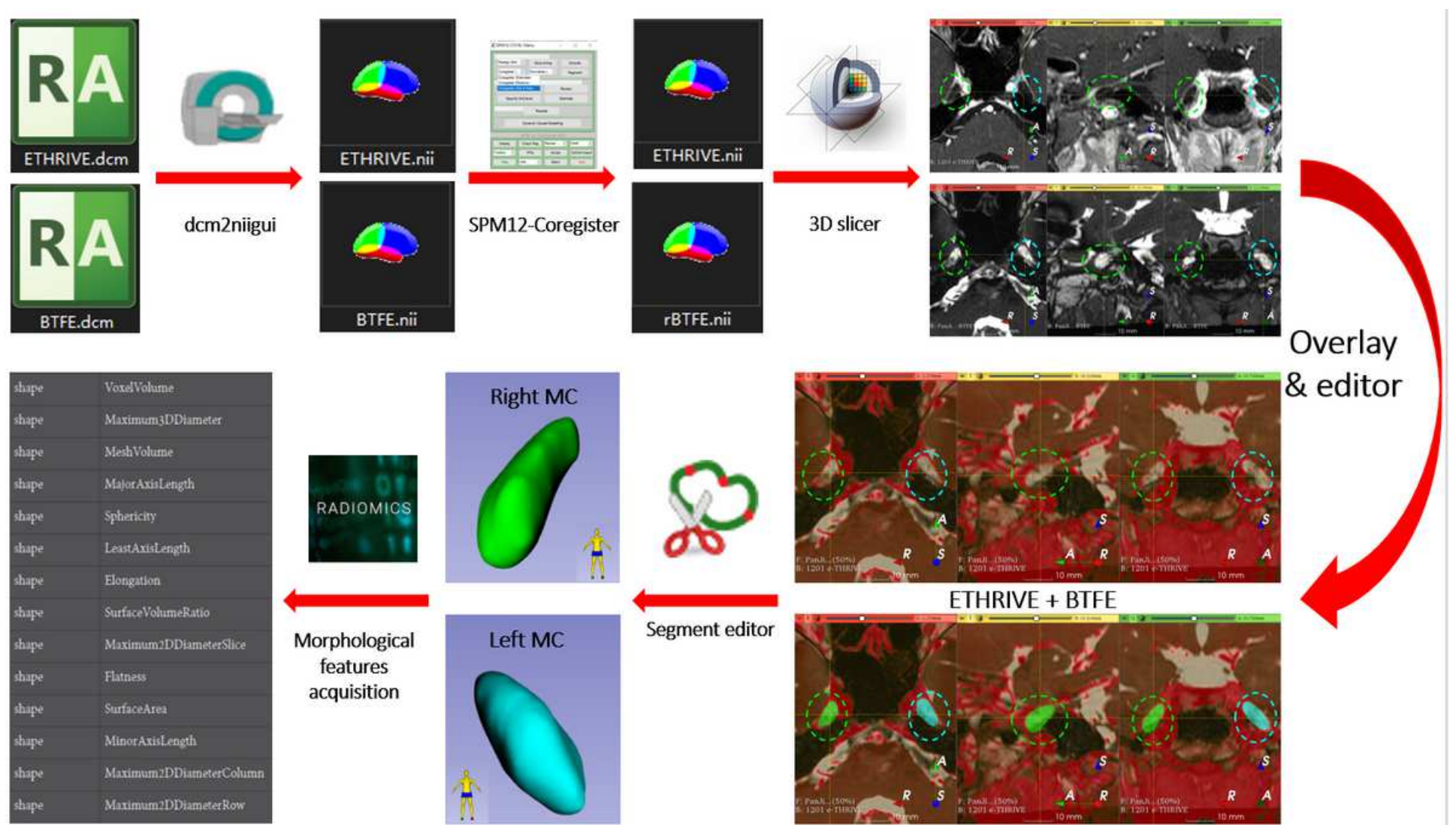

Figure 1

Morphological data acquisition flowchart 

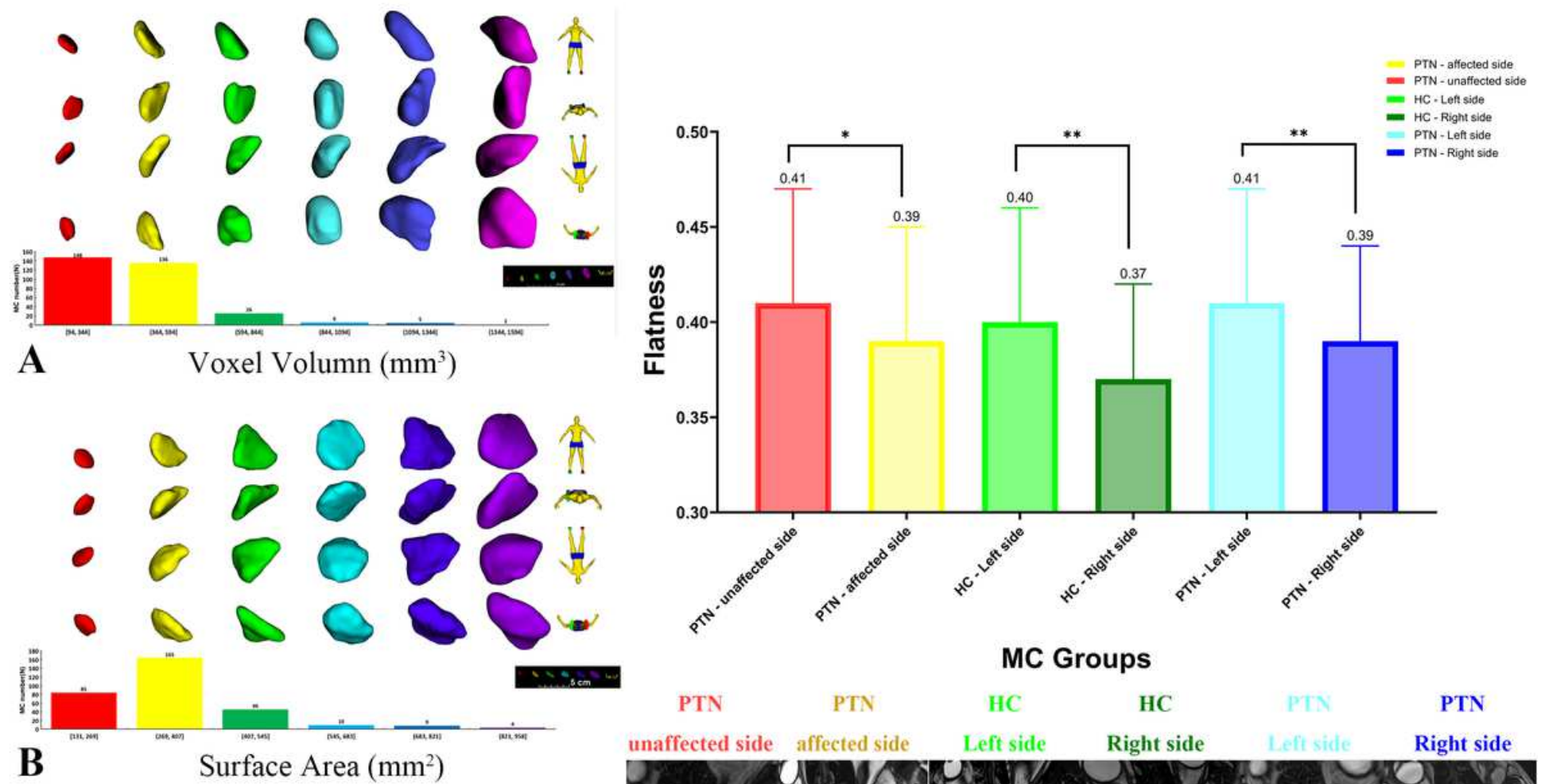

\section{Groups}

PTN PTN HC HC PTN

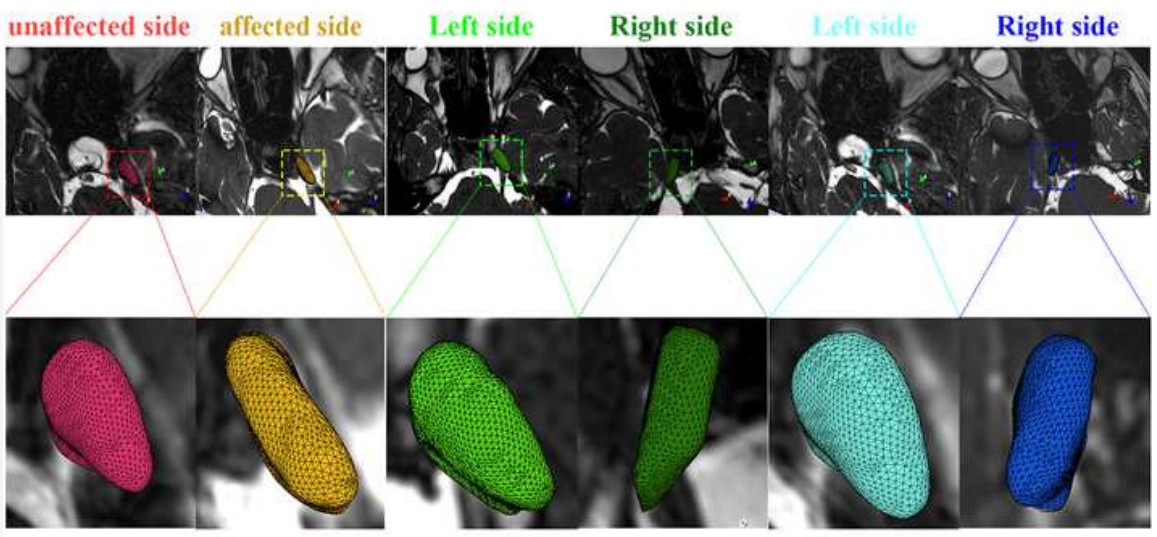

D The Flatness of MC was compared between different groups.

Figure 2

Morphology of MC with different morphological characteristics. (A) The red MC's voxel volume is $94 \mathrm{~mm} 3$, the yellow is $347 \mathrm{~mm} 3$, the green is $601 \mathrm{~mm} 3$, the light blue is $904 \mathrm{~mm} 3$, the navy blue is $1136 \mathrm{~mm} 3$ and the purple is $1589 \mathrm{~mm} 3$. (B) The red MC's surface area is $131 \mathrm{~mm} 2$, the yellow is $335 \mathrm{~mm} 2$, the green is $482 \mathrm{~mm} 2$, the light blue is $569 \mathrm{~mm} 2$, the navy blue is $784 \mathrm{~mm} 2$ and the purple is $853 \mathrm{~mm} 2$. (C) The red MC's flatness is 0.25 , the yellow is 0.32 , the green is 0.37 , the light blue is 0.42 , the navy blue is 0.47 and the purple is 0.54. (D) The flatness of MC was compared between different groups. ${ }^{*} P<0.01 \rrbracket * P<0.05$; 

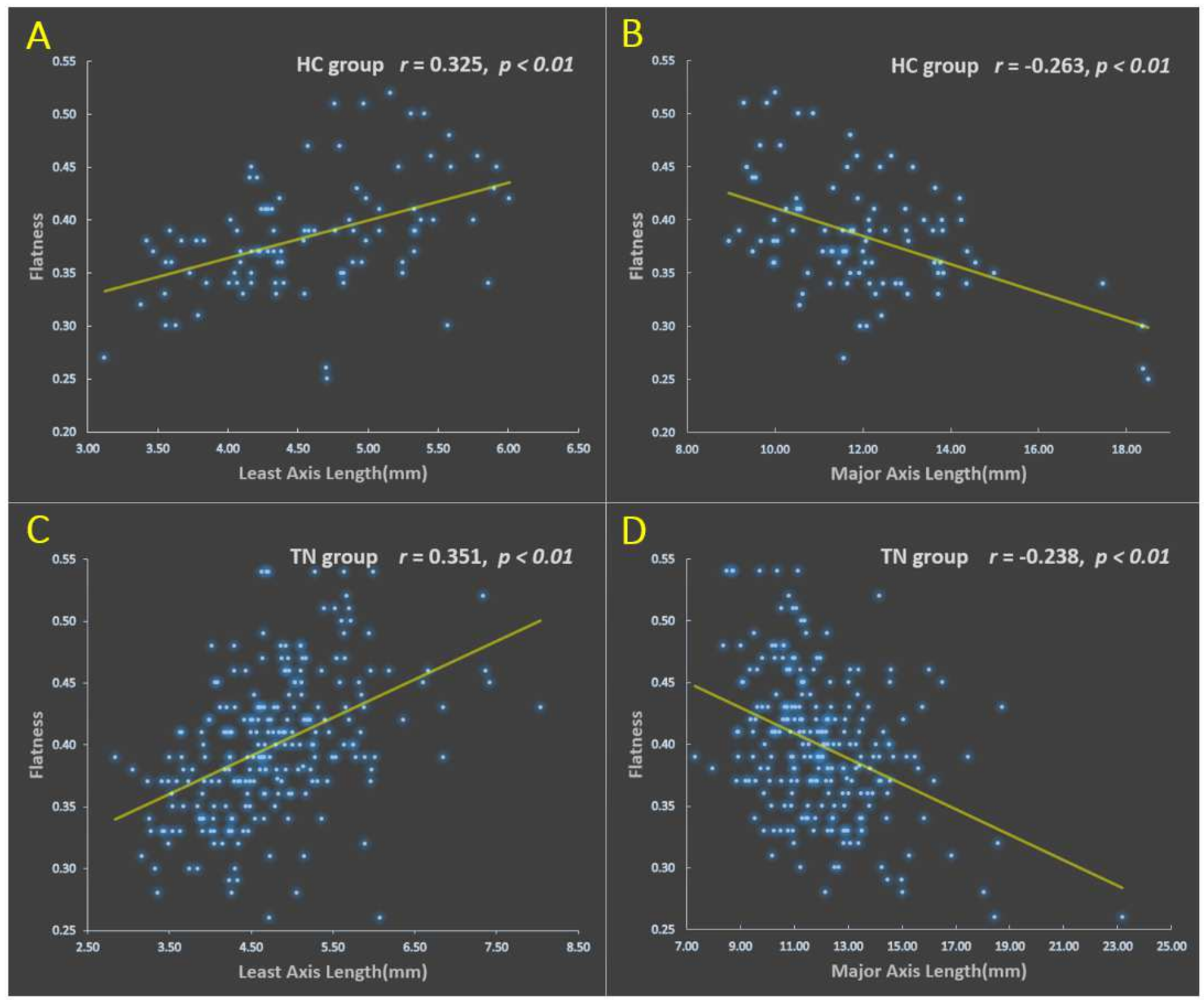

\section{Figure 3}

Scatter plot of correlation coefficient of flatness. (A) Scatter plots of the flatness positively correlated with the least axis length in the $\mathrm{HC}$ group $(r=0.325, P<0.01)$. (B) Scatter plots of the flatness negatively correlated with the major axis length in the HC group $(r=-0.263, P<0.01)$. (C) Scatter plots of the flatness positively correlated with the least axis length in the TN group $(r=0.351, P<0.01)$. (D) Scatter plots of the flatness negatively correlated with the major axis length in the TN group $(r=-0.238, P<0.01)$. 

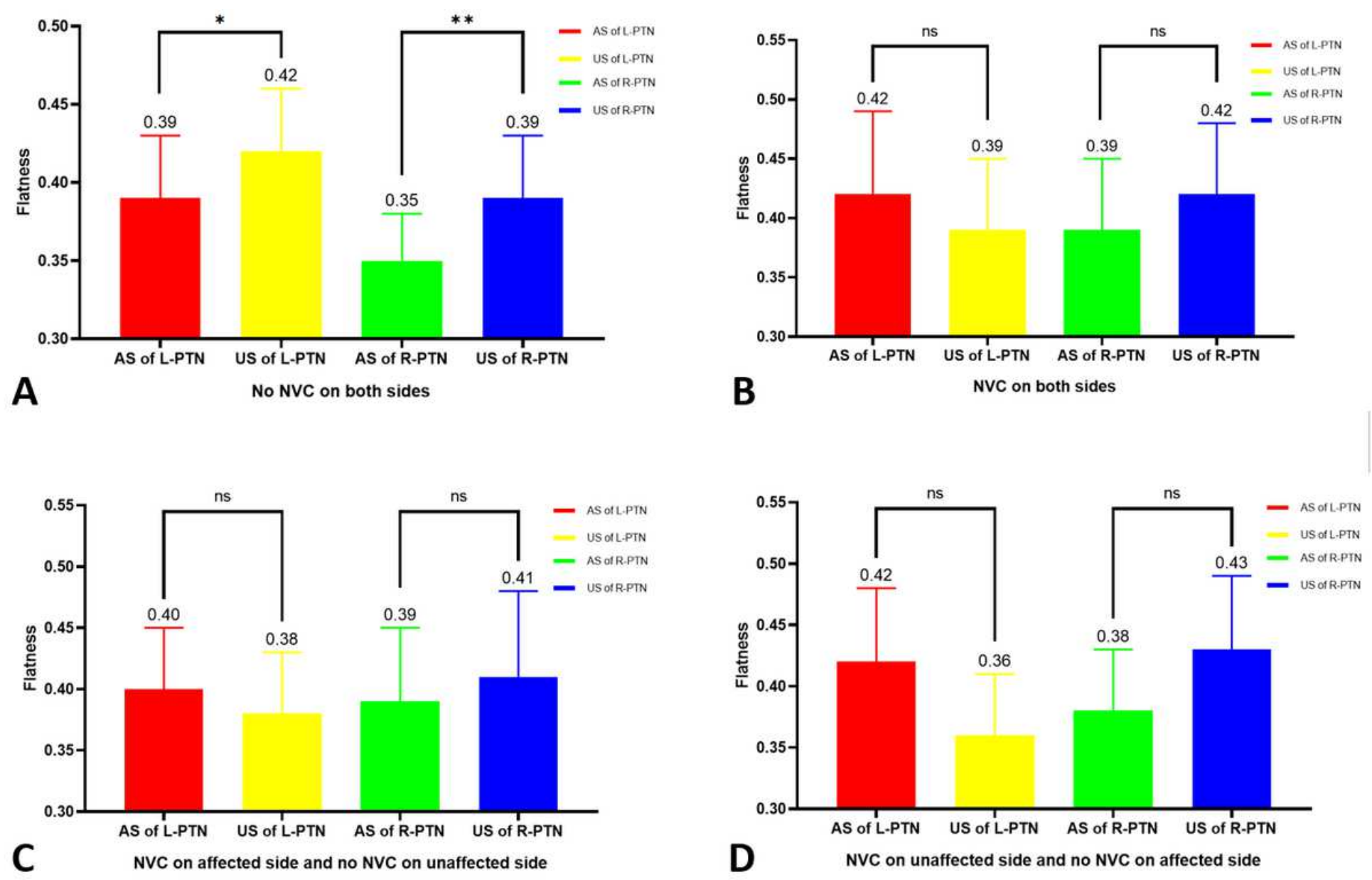

Figure 4

Comparative analysis of Flatness with bilateral MCs under different NVC conditions. (A) No NVC on both sides. The MC of the affected side of the left PTN without bilateral NVC was lower than that of the unaffected side $(0.39 \pm 0.04$ vs $0.42 \pm 0.04, P<0.051)$, the $M C$ of the affected side of the right PTN without bilateral NVC was lower than that of the unaffected side $(0.35 \pm 0.03$ vs $0.39 \pm 0.04, P<0.05)$. (B) NVC on both sides. There was no significant difference between groups. (C) NVC on affected side and no NVC on unaffected side. There was no significant difference between groups. (D) NVC on unaffected side and no NVC on affected side. There was no significant difference between groups. ${ }^{*} P<0.051$; $* \star P<0.05$; ns $\mathbb{X}$ > 0.05; NVC: neurovascular contact; L: Left; R: Right; AS: Affected side; US: Unaffected side. 